It is over twenty years since epidemiological studies revealed that there was a relationship between patterns of early growth and risk of developing type 2 diabetes in later life. Studies of identical twins, individuals who were in utero during periods of famine and animal models have provided strong evidence that the early environment, including early nutrition, plays an important role in mediating this relationship. The concept of "early life programming" is therefore widely accepted. However the mechanisms by which a phenomenon that occurs in early life can have long-term effects on the function of a cell and therefore metabolism of an organism many years later are still emerging.

These include:

1. Permanent structural changes in an organ due to exposure to suboptimal levels of essential hormones or nutrients.

2. Permanent effects on regulation of cellular ageing through increases in oxidative stress and mitochondrial dysfunction leading to DNA damage and telomere shortening.

3. Persistent alterations in epigenetic modifications (including DNA methylation, histone modifications and miRNAs) leading to changes in gene expression.

Several transcription factors have been shown to be susceptible to programmed changes in gene expression through such epigenetic mechanisms. These are conceptually attractive targets of programmed epigenetic regulation, as through regulation of their expression a network of other genes will be regulated. Further understanding of the extent and nature of these programming mechanisms could enable the development of preventative and intervention strategies to combat the burden of diseases such as type 2 diabetes.

\section{THE ROLE OF FGF10 FOR ALVEOGENESIS IN BRONCHOPULMONARY DYSPLASIA}

doi:10.1136/archdischild-2012-302724.0176

${ }^{1} \mathrm{CM}$ Chao, ${ }^{2} \mathrm{D}$ Al Alam, ${ }^{3} \mathrm{C}$ Tiozzo, ${ }^{4} \mathrm{R}$ 5Virender, S Bellusci. ${ }^{1}$ Department of Pediatrics, Excellence Cluster in Cardio-Pulmonary Systems (ECCPS), University of Gießen, Giessen, Germany; ${ }^{2}$ Developmental Biology and Regenerative Medicine Program, Saban Research Institute of Children's Hospital; ' ${ }^{3}$ Developmental Biology Program, Division of

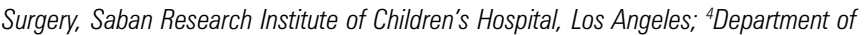
Pediatrics, Los Angeles Biomedical Research Institute at Harbor-UCLA Medical Center, Torrance, CA, USA; ${ }^{5}$ Department of Internal Medicine II, Excellence Cluster in CardioPulmonary Systems (ECCPS), University of Gießen, Giessen, Germany

Background and aim Bronchopulmonary dysplasia (BPD) is associated with impaired alveolar growth and pathologic vascularization. As a chronic lung disease it remains an important complication for preterm infants, especially born before 28th week of gestational age. Fibroblast-growth-factor 10 (Fgf10) is known to play an important role in lung morphogenesis. We aim to investigate the role of Fgf10 for alveogenesis in a mouse model of BPD.

Methods Using an inducible double transgenic mouse line (SPC-rtTA; tet $(O) F g f 10)$ we established a BPD model by exposing the pups to $85 \%$ oxygen (experimental and control group) and normoxia (control group) for 28 days. Activation of the transgene Fgf10 was done after birth (P0) by doxycycline treatment. Gene expressions for Fgfr2b and Fgf10 were analyzed by quantitative real-time PCR. To study lung morphology histology, mean linear intercept (MLI) and radial alveolar count (RAC) were performed.

Results Real-time PCR results showed a significant decrease of Fgfr $2 b$ and Fgf 10 expression in the hyperoxia group at day 21 and day 28 indicating epithelial cell damages. The histology showed a simplification of alveoli in the hyperoxia group ( $85 \%$ oxygen) but not in the normoxia group after 14 days. In contrast, the hyperoxia group with overexpression of Fgf 10 showed less simplification of alveoli. These findings were confirmed by MLI and RAC.
Conclusion We conclude that Fgf10 may have a protective/regenerative effect on lung injury by increasing secondary septa formation.

\section{AUTOMATION OF RESPIRATORY SUPPORT IN THE NEONATE: FACT OR FICTION}

doi:10.1136/archdischild-2012-302724.0177

E Bancalari. Pediatrics/Neonatology, University of Miami Miller School of Medicine, Miami, FL, USA

Premature infants frequently present with respiratory instability that is associated with fluctuations in ventilation and gas exchange. Frequent adjustments of respiratory support to match the infant's needs are time consuming and are limited by staff availability and workload. Hence, automation is being developed as a way of improving the care of the premature infants and reduce staff workload.

Some of these automated modes of respiratory support are becoming available for clinical use in preterm infants. These include volume targeted ventilation where peak inspiratory pressure is automatically and continuously adjusted to deliver a preset tidal volume. Another modality is targeted minute ventilation where the ventilator rate is adjusted automatically to maintain a preset minute ventilation. Proportional assist ventilation is another modality where airway pressure is adjusted in proportion to flow or tidal volume generated by the infant. Using this principle recently NAVA has been introduced for use in neonates where the airway pressure generated by the ventilator is proportional to the electrical signal captured from the diaphragm. Finally, automated adjustment of inspired oxygen concentration is becoming available in some ventilators to adjust $\mathrm{FiO} 2$ and maintain oxygen saturation within a preset range. These modes are expected to compensate for some of the limitations that exist in the present forms of respiratory support. Available evidence and preliminary findings for short term effects are promising but further investigation is needed to determine the effects of these modalities on the long term outcome of preterm infants.

\section{CIRCUMSTANCES SURROUNDING END OF LIFE OF INFANTS WITH PERINATAL HYPOXIC-ISCHEMIC ENCEPHALOPATHY (HIE)}

doi:10.1136/archdischild-2012-302724.0178

${ }^{1} \mathrm{~A}$ Garcia-Alix, ${ }^{2} \mathrm{~J}$ Arnaez, ' $\mathrm{V}$ Cortes, ${ }^{3} \mathrm{G}$ Arca, ${ }^{1} \mathrm{~N}$ Herranz, ${ }^{4} \mathrm{~F}$ Gaya, ${ }^{5} \mathrm{~A}$ Balaguer. ${ }^{1}$ Division of Neonatology, Sant Joan de Deu Hospital. University of Barcelona, Esplugues de Llobregat; '2Division of Neonatology, Hospital Universitario Burgos, Burgos; ${ }^{3}$ Division of Neonatology, Hospital Clinic-Maternitat, Barcelona; "Division of Bioestatistics, Hospital Universitario La Paz, Madrid; ${ }^{5}$ Pediatrics, International University of Barcelona, Barcelona, Spain

Objective To analyze circumstances of all consecutive neonatal deaths by HIE over a 10 year period and examine changes along timeSetting. Level III Neonatal Intensive Care. Madrid, Spain.

Design Retrospective chart review of all neonatal cases with HIE who died from 2000 to 2010 within the neonatal period.

Results Of a total 70 infants with HIE, 18 died during the neonatal period. All of them had severe HIE and the mean age of death was $64.4 \pm 51$ hours of life. In 17 (94\%) the death was preceded by an end of-life decision based on the bad prognosis; 15 by withdrawal or limitation of therapy (W/LT) while ventilated, and 2 by decision of parents not to resuscitate if cardiac arrest. All patients had coma and at least one of the following studies severely altered: EEG-aEEG, US/Doppler or CSF-NSE. The first interview for W/LT happened at $25.7 \pm 28.9$ hours and the mean time interval since $\mathrm{W} / \mathrm{LT}$ was iniciated until death was $10.5 \pm 14$ hours. Ten infants $(56 \%)$ had sedation or analgesia during W/LT and presence of the family at the bedside 